
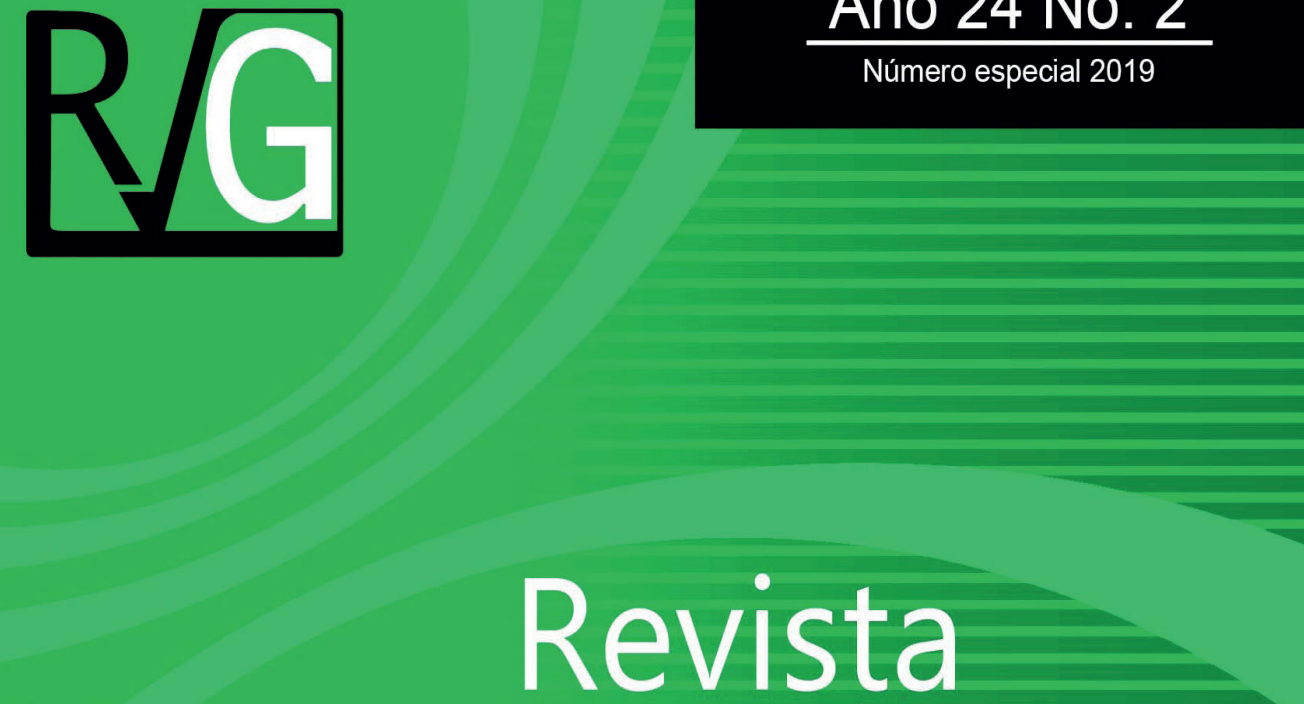

Venezolana de

$\frac{1}{6}$

$\frac{\mathbb{O}}{\frac{1}{0}}$
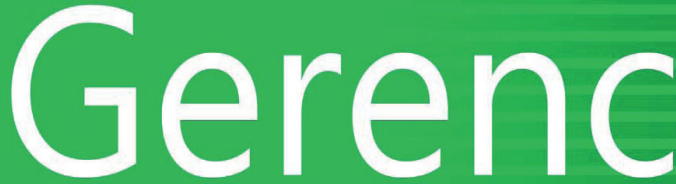

0

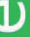

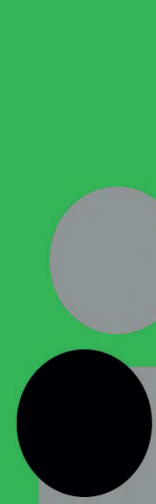

ro

u

$>$

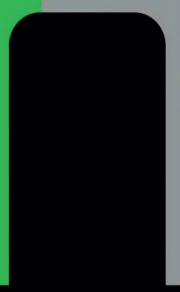




\title{
Gestión de calidad para la proyección socio- cultural y extensión universitaria
}

\author{
Paredes López, Lilian Roxana ${ }^{1}$ \\ Curo Maquén, Luis Alberto ${ }^{2}$ \\ Carbajal Cornejo, Katherine ${ }^{3}$ \\ Núñez Puse, Sonia Magali ${ }^{4}$
}

\section{Resumen}

Las universidades públicas atienden a normativas oficiales, emanadas de organismos gubernamentales para la gestión interna de los procesos; siendo beneficioso que se apliquen mecanismos eficientes para la mejora continua y por ende para el logro de la calidad en la gestión. En el caso de estudio el modelo EFQM proveerá información para el logro del objetivo general que es: proponer un plan de mejoramiento de la gestión de la Oficina de Proyección Social, Extensión Cultural y Universitaria (OPSECU), de la Facultad de Ciencias Físicas y Matemáticas (FACFyM), de la Universidad Nacional Pedro Ruíz Gallo. La investigación es de campo, tipo propositiva, no experimental de corte transversal. La población estuvo conformada por 1926 estudiantes y 176 docentes. La recolección de datos se hizo con la técnica de la encuesta, con instrumentos respectivamente validados y para el procesamiento de los datos, el software SPSS-23.

Recibido: 11-09-19 Aceptado: 10-11-19

Doctora en Ciencias de la Educación, Magister en Docencia Universitaria e Investigación Educativa. Licenciada en estadística y Licenciada en Educación. Universidad Nacional Pedro Ruiz Gallo-UNPRG. ORCID: 0000-0002-4290-1216. Email: Iparedes@unprg.edu.pe

2 Doctor en Ciencias de la Educación, Magister en Docencia Universitaria e Investigación Educativa. Licenciado en Física y Licenciado en Educación. Universidad Nacional Pedro Ruiz Gallo. ORCID: 00000002-1298-7186. Email: Icuro@unprg.edu.pe

3 Doctora en Educación, Magister en Gestión y Docencia Educativa, Segunda Especialidad en Gestión Educativa, Segunda Especialidad en Comunicación y Matemática para el II ciclo de la E.B.R. Licenciada en Educación Inicial. Universidad Santo Toribio de Mogrovejo. ORCI: 0000-0003-3339-9217. Email: kcarbajal@ usat.edu.pe

4 Magíster en Docencia Universitaria y Gerencia Educativa, Licenciada en Administración de Empresas con más de 13 años en E.S. Experiencia metodológica y temática en asesoramiento de tesis. Universidad César Vallejo. ORCID: 000-0001-9648-8108. Email: snunezpm@ucvvirtual.edu.pe 
Se evidencia deficiencia en la gestión de la oficina de proyección social y extensión universitaria, además desvinculación de la universidad con el entorno en los aspectos relacionados con esas actividades. Se concluye que un plan de mejoramiento transformará la gestión de la OPSECU hacia la calidad total.

Palabras clave: gestión de calidad; proyección social; extensión cultural y universitaria; Modelo de excelencia EFQM; Facultad de Ciencias Físicas y Matemáticas.

\title{
Quality management for socio-cultural projection and university extension
}

\begin{abstract}
Public universities attend oficial regulations, emanating from government agencies, for internal process management; it is beneficial that efficient mechanisms be applied for continuous improvement and therefore for the achievement of quality in management. In the case of study, the EFQM model will provide the information for achievement of the general objective that is: to propose a plan to improve the management of the Office of Social Projection, Cultural and University Extension of the Faculty of Physical and Mathematical Sciences of the National University Pedro Ruíz Gallo. The research is field, purposeful non-experimental, cross sectional. The population was made up of 1.926 students and 176 teachers. The data collection was done with the survey technique, with respectively validated instruments and for data processing the SPSS-23 software. There is evidence of deficiency in the management of the office of social projection nd university extensión, as well as disconnection of the university with the environment in aspect related to these activities. It is concluded that an improvement plan will transform the management of OPSECU towards total quality.
\end{abstract}

Keywords: Quality Management, social projection, cultural and university extensión, EFQM Model of Excellence, Faculty of Physical Sciences and Mathematics.

\section{Introducción.}

Hasta hace muy poco tiempo, la gestión de calidad en las instituciones educativas, especialmente universitarias, no era un tema prioritario para ser incluido en los planes estratégicos; de hecho los gobiernos solo daban prioridad al financiamiento de los aspectos básicos, digamos los necesarios para el funcionamiento o la operatividad, como son: infraestructura, edificaciones, tecnología, programas, currículo, sueldos, etc.; sin hacer parte a las instituciones educativas de procesos de transformación que signifiquen aumentar el nivel, en aspectos relacionados con la aplicación de técnicas para elevar la 
calidad, y por ende atender las posibles deficiencias que evitan cumplir con algunas exigencias del exterior, pero también del interior de sus estructura.

En su investigación demuestra científicamente como Instituciones de Educación Superior pueden orientar su gestión hacia resultados de excelencia, cuando implementan modelos asociados a los sistemas de gestión de la calidad. (Calvo, et al., 2005; citado por Fontalvo y De La Hoz, 2018). En la actualidad existen nuevas exigencias y condiciones generadas por la innovación y las nuevas tecnologías de la información, esto en primer lugar; en segundo lugar, los mismos procesos que se ejecutan organizativamente inducen y reconducen las acciones, inevitablemente hacia la innovación; requiriendo para ello que las personas encargadas de realizar esas acciones, los líderes, los directores, sean de una institución educativa o de una empresa, en conjunto con los equipos de trabajo y personal a su cargo, sean capaces de responder a esas exigencias y necesidades del entorno (clientes, sociedad).

En ese sentido la implantación de un sistema de gestión de calidad, ajustado a normas y aplicado a procesos y procedimientos claves, será un apoyo fundamental para el direccionamiento estratégico que las instituciones necesitan. (Fontalvo, et al., 2018). Lo anteriormente expuesto, justifica la realización de esta investigación, en el sentido que, una propuesta de gestión de calidad, de la proyección social, extensión cultural y universitariafortalecerá la función académica, promoverá el impacto social que se necesita; así como también, impactará a la organización positivamente, puesto que se elevará el nivel de calidad y excelencia de la gestión y por ende en los resultados por los procedimientos y actividades realizadas.

Lo antes expuesto, da paso al planteo del objeto del estudio, que se persigue: "Proponer un modelo de gestión para la - Proyección Social, Extensión Cultural y Universitaria- de la Facultad de Ciencias Físicas y Matemáticas de la Universidad Nacional Pedro Ruiz Gallo"; basado en el Modelo de excelencia EFQM que permitirá orientarles en la comprensión de las dificultades existentes y estimulando la búsqueda de soluciones. La población, estuvo constituida por todos los docentes adscritos a la FACFyM en el semestre académico 2015-I y por todos los estudiantes de la FACFyM, matriculados en el semestre académico 2015-I.

La muestra que participó en la investigación, fue seleccionada por medio de un muestreo aleatorio estratificado, considerando como estratos las 5 escuelas profesionales: Computación, Ingeniería Electrónica, Estadística, Física y Matemática, para docentes y estudiantes; y para los egresados y exjefes, fue un muestreo no probabilístico, por conveniencia, quedando conformada por 248 individuos. En cuanto a los Docentes de la muestra: $80 \%$ son de sexo masculino y $20 \%$ de sexo femenino. En cuanto a la especialidad el $20 \%$ son de Computación, $16,7 \%$ son de Ingeniería Electrónica, 13,3\% de Estadística, 16,7\% de Física y 33,3\% de Matemáticas. Los estudiantes que conformaron la muestra, $63,8 \%$ son de sexo masculino y el $36,2 \%$ son de sexo femenino; en cuanto a la especialidad, el $26,6 \%$ son de: Computación; $25,7 \%$ son de Ingeniería Electrónica; $21,1 \%$ son la especialidad de Estadística, $11 \%$ de Física y $15,6 \%$ de Matemáticas.

El instrumento utilizado fue 
el cuestionario, constituido por 16 preguntas con escalamiento Likert, definiendo cinco opciones (Nunca, Casi nunca, A veces, Casi siempre, Siempre). De las cuales solo se seleccionaron 10 a criterio e intereses de los investigadores de este artículo. Se probó la confiabilidad y validez del cuestionario: Confiabilidad Alfa de Cronbach, basado en elementos estandarizados $\alpha=0.912, \quad L a$ validez para el cuestionario arrojó $\mathrm{KMO}=0.875$, la prueba de esfericidad es menor a 0.05 ; así mismo las comunalidades son mayores a 0.4 que es lo establecido.

\section{Algunas definiciones sobre Calidad y Calidad en educación.}

Al respecto, Alvarado, Hernández y Chumaceiro, (2010), tomando en consideración la definición planteada por: Ferrando y Granero (2008:13), señalan sobre el concepto de calidad que "esta ha existido desde que el hombre ha existido... desde los tiempos en que el trueque era práctica habitual hasta nuestros días, pues siempre que adquirimos algún producto tenemos unas expectativas". Por ello, hablar de calidad es remitirnos a una serie de posturas subjetivas que, en la mayoría de las ocasiones, establecen definiciones con base en su objeto y ocasionalmente en su origen, (Alvarado e Izaguirre, 2015).

Es muy importante destacar para los efectos de este estudio, que cuando se habla de calidad, nos referimos a la calidad educativa; pero como bien lo expresan, Martínez, Pérez y Martínez, (2016), "la calidad es un constructo que nace vinculado al concepto de "kaizen" (cambio para mejorar o mejora continua) y que determina unos principios que sirven para establecer un plan de acción a partir del cual una organización, en nuestro caso la universidad. La anterior definición se puede ajustar perfectamente al objetivo de esta investigación, sin embargo; en torno al término "calidad", la naturaleza y fines de las instituciones educativas requieren de un tratamiento específico, aunque se empleen modelos que se han originado dentro de empresas o para estas.

Si bien es cierto, que los resultados del manejo adecuado de las instituciones educativas aplicando las normativas y modelos de gestión de calidad que se sugieren, las convierten en una suerte de "empresas educativas"; y han dado resultados beneficiosos, aportando valor a las mismas, observándose además la mejora continua en muchos procesos y aspectos en su funcionamiento; aun así, no es equivocado decir que también se les ha dado un tinte mercantilista, criterio este no muy beneficiosos para los "clientes" que vienen siendo los estudiantes. Tal como lo indica, Pulido y Espinoza (2018), citando a: Escudero, González y Rodríguez (2013), quienes alertan sobre la concepción de calidad, la cual es adaptable a una reserva de minorías selectas; y a: Brown y Carasso (2013), quienes señalan que la estrategia de la mejora de la calidad de los estudiantes a través de la mercantilización, obliga a las universidades a competir por el alumnado.

"En el ámbito educativo, especialmente en educación superior, la calidad es uno de los mensajes y objetivos más repetidos, con cierto consenso social en torno al mismo, incluso varias normativas lo han incluido en su denominación (Ley de Calidad de la Educación, 2012 y Ley para la Mejora de la Calidad Educativa, 2013)", cita de Martínez, et al., (2016).

Por su parte, González, (1990:31); 
analizado por Pulido, et al., (2018), ha trabajado en el tema, postulando que "el concepto de calidad en la educación superior no existe como tal, sino como un término de referencia de carácter comparativo en el cual algo puede ser mejor o peor que otro, dentro de un conjunto de elementos homologables, o en comparación con cierto patrón de referencia -real o utópico- previamente determinado". Por lo tanto, solo se debe plantear que una institución es mejor que otra cuando son homólogas en sus fines, es decir que tengan similitud en su misión, naturaleza o funciones y el entorno donde se desenvuelvan.

En otro orden de ideas, haciendo alusión a la calidad total, para elevar los niveles de esta en la gestión de la Proyección socio-cultural y extensión universitaria, en la Facultad de Ciencias Físicas y Matemáticas de la Universidad Pedro Ruíz Gallo, se debe aplicar un sistema de gestión de calidad (SGC) que permita, tal como se expresó anteriormente, seleccionar criterios que les oriente de forma práctica y segura hacia el logro del fin, como lo es lograr la amplitud y coordinación de procesos en la OPSECU de esa facultad, para la vinculación de la universidad con el entorno circundante.

\section{Sistema de Gestión de Calidad (SGC): Generalidades.}

Tal como se ha planteado hasta ahora la implementación del término "calidad", analizado como un criterio importante para la mejora de los procesos, en relación al contexto del estudio, el cual es una universidad pública; el tipo de gestión que se lleve a cabo, tomando en cuenta esta perspectiva debe estar orientada a realizar una eficaz administración de los recursos del Estado, con el objeto de satisfacer las necesidades de la sociedad y promover el desarrollo de la región, según parámetros y normativas establecidos por los entes gubernamentales. De la misma manera, realizar una gestión social, por medio de la cual se apliquen una serie de mecanismos que fomenten la inclusión social y los nexos entre la universidad y la comunidad donde se encuentra inmersa la institución, esto último se evidenciaría en los proyectos sociales que se puedan ejecutar. (Paredes y Curo, 2019)

Los anteriores, son dos tipos de gestión que de acuerdo a la naturaleza de la organización, deberían dirigir y administrar en pro de resolver situaciones; tomando el tiempo y el presupuesto disponible, empleando un conjunto de operaciones, procesos y acciones, ejecutados para el cumplimiento de metas y objetivos, a través de un ciclo sistémico y continúo. La intención debe ser, gestionar de buena manera los recursos, para lograr las metas y planes que hayan trazado las instituciones. Partiendo de esa premisa, una gestión bien llevada, supone el manejo de procesos de evaluación interna y externa, procesos de autoevaluación, mejoramiento progresivo, etc. Pero además conlleva un seguimiento continuo de estos procesos, el manejo de recursos, así como, la incorporación de medidas remediales en caso de ser necesario (Bilen, 2010; Pratasavitskaya y Stensaker, 2010; Manatos, Sarrico y Rosa, 2017; citados por Pulido, et al., 2018).

En la actualidad, los niveles de competitividad y de globalización aplicables a casi todos los sectores, han provocado un amplio desarrollo del concepto de gestión de calidad, como un enfoque estratégico para el tratamiento 
de la calidad en la empresa, que se sustenta en los principios de orientación al cliente, mejora continua, enfoque en las personas y visión global de la organización (Camisón et al., 2009; citado por Castillo, Mercado, Prado, Soto, 2014).

En este entorno, aparecen los sistemas de gestión de la calidad (SGC), que permiten a las empresas dotarse de sistemas de gestión para establecer políticas y responsabilidades, asignar recursos, e identificar actividades clave (Criado y Calvo, 2009; citado por Castillo et al., 2014)

El significado de un Sistema de Gestión de Calidad (SGC) es de resaltar en este estudio, en vista de la importancia que tiene su implementación para la gestión desarrollada en la OPSECU y los efectos que tiene en la toma de decisiones para la concreción de los fines institucionales; todo lo antes dicho depende de la capacidad de dirección, organización, planificación que posean los directores y personal. La adopción de un SGC, es una decisión de carácter estratégico para optimizar los indicadores de desempeño, frente a una realidad institucional prexistente.

Dicho lo anterior, uno de los ejes principales de esta investigación es, orientar la aplicación de procedimientos para el desempeño eficiente de las funciones de la proyección social-cultural y extensión universitaria (OPSECU), por medio del servicio y las acciones directas sobre diversas situaciones del entorno donde hace vida la universidad, con la intención de solucionar problemas existentes. Para ello, como bien se sabe, la interacción entre la universidad y estos sectores debe estar enmarcada por directrices que sean efectivas $y$ eficientes, lo cual viene dado por el desarrollo de una gestión de calidad, como ya se ha planteado.

En el caso de la Facultad de Ciencias Físicas y Matemáticas (FCFyM), de la "Universidad Pedro Ruíz Gallo", y la Oficina de Proyección Social y Extensión Cultural y Universitaria (OPSECU), se analizaron algunos indicadores con la intensión de caracterizar la gestión, básicamente realizar una autoevaluación, recopilar la información necesaria para luego realizar las respectivas reflexiones y los aportes pertinentes, tendientes a remediar las fallas existentes y fortalecer la estructura interna de este ente. Siendo necesario también el compromiso de todos y cada uno de los involucrados en este proceso. Para ello se ha seleccionado el Modelo de Gestión de Calidad EFQM, como apoyo al direccionamiento estratégico del funcionamiento de la OPSECU; el mismo será analizado en el siguiente apartado de este artículo.

\section{Modelo de Excelencia de la European Foundation for Quality Management (EFQM)}

EI Modelo EFQM de excelencia es de propiedad intelectual de la European Foundation for Quality Management, en la actualidad es el modelo más utilizado; puesto que independientemente del sector, tamaño, estructura o madurez institucional; las organizaciones necesitan establecer sistemas apropiados de gestión. El Modelo de excelencia de la EFQM, viene siendo esa herramienta práctica para ayudar a las organizaciones en su camino hacia la excelencia, orientándoles en la comprensión de las dificultades existentes y estimulando la búsqueda de soluciones. Actualmente es el modelo más utilizado para la evaluación de 
la excelencia por las organizaciones empresariales europeas. (Paredes, et al., 2019).

Tal como lo describe, Martínez et al., (2016), "Es un modelo flexible que puede aplicarse a cualquier tipo de organización, con independencia de su tamaño o sector (público o privado)". Emplea como método la orientación al cliente y el enfoque basado en procesos. Para su adecuado funcionamiento, necesitan de un compromiso claro y decidido por parte de la dirección de la empresa.

Una breve descripción del Modelo EFQM es como sigue: se centra en los criterios de gestión (liderazgo, estrategia, personas, alianzas $y$ recursos, procesos, resultados) y su planificación, despliegue y evaluación. No es normativo y no se puede obtener ningún certificado que acredite su aplicación, se basa en la autoevaluación mediante un sistema de ponderación de los diversos criterios prefijados. Aporta una estructura sistemática de análisis que permite a las organizaciones revisar su sistema de gestión y comprobar su avance hacia la excelencia; se orienta al cliente y al logro de resultados, tiene una visión más amplia al incorporar también los resultados que afectan a los diversos grupos de interés de la organización: agentes, clientes, personas, socios, sociedad, etc. (Martínez, et al., 2016).

Las organizaciones utilizan el Modelo de Excelencia de la EFQM, como fundamento para la operativización de las metas a conseguir, desde la planificación, realización, autoevaluación y revisión de sus proyectos. Por lo tanto, este modelo es una herramienta, que sirve para: Comparar las mejores prácticas entre organizaciones. - Guía para identificar las áreas de mejora.- Base para un vocabulario y estilo de pensamiento común. - Estructura para los sistemas de gestión de las organizaciones, (Paredes et al., 2019).

Ahora bien el Modelo de Excelencia de la EFQM es un marco no prescriptivo basado en nueve criterios, cinco de ellos "Facilitadores o agentes" y cuatro "Resultados". Los criterios "Agentes" se refieren a lo que una organización hace. Los criterios "Resultados" se relacionan con los que una organización logra. Los resultados son causados por los "Agentes" y se mejoran mediante la retroalimentación. Se puede observar, en el siguiente diagrama, las relaciones de los criterios en el Modelo. Las flechas enfatizan la naturaleza dinámica del modelo. Muestran la innovación y el aprendizaje organizacional para mejorar los agentes que a su vez llevan a la mejora de los resultados (Paredes et al., 2019)

En definitiva el Modelo EFQM es un modelo de Gestión de Calidad Total desde su creación, que se ha desarrollado para materializar los principios de excelencia. Se basa en conseguir la satisfacción de clientes y empleados, además del impacto que provoca en la sociedad; implementarlo con éxito se consigue mediante un liderazgo que impulse la política y las estrategias, a las personas de la organización, establezca alianzas, administre recursos y los procesos hacia la consecución de la excelencia en los resultados (Martínez et al., 2016).

El Modelo EFQM, puede implementarse a situaciones problemáticas del ámbito educativo, tener conocimiento de la misma por medio de la autoevaluación y reflexión de la información recabada; con este insumo se podrá elaborar y proponer planes y estrategias de mejora continua (Autoevaluaciones periódicas), para 


\section{Figura 1. \\ Criterios del Modelo de Excelencia EFQM}

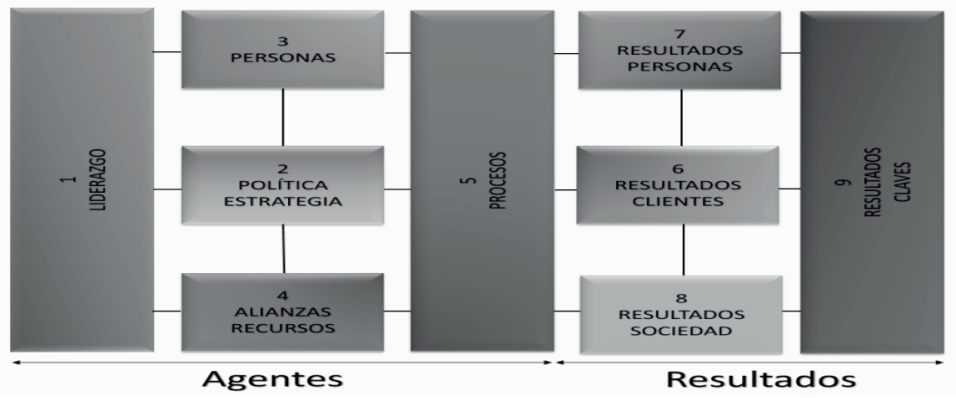

Fuente: Gómez (2017).

ello es necesario el compromiso de todos y cada uno de los involucrados en el proceso; lo que conllevaría a la participación en la toma de decisiones, aplicación de directrices y realización de las tareas pertinentes al proceso de la elevación de la calidad de los servicios que brinda la Institución educativa.

El Modelo EFQM se basa en unos principios, los cuales de adoptarse a modo de guía podrían generar el logro de resultados de excelencia y por consiguiente de la calidad; y son los siguientes: - Orientación hacia los resultados - Orientación hacia el cliente - Gestión por procesos y hechos - Liderazgo y perseverancia en los objetivos - Desarrollo e implicación de las personas - Aprendizaje, innovación y mejora continua - Desarrollo de alianzas - Responsabilidad Social. (Gómez, 2017).

\section{Principios de la calidad total del Modelo EFQM: Aplicación en la "Oficina de Proyección social y extensión cultural y universitaria" OPSECU:}

Estos principios adaptados o interpretados de manera coherente, permiten tener conocimiento de cómo está funcionando la institución; con el fin de elaborar proyectos y planes de mejora de acuerdo con las carencias deficiencias o necesidades detectadas por medio de la autoevaluación que se realice en la institución. En este sentido, en el siguiente cuadro (Cuadro 1), se muestra un análisis y adaptación, tomando en cuenta los principios del modelo (EFQM), con la finalidad de orientar sobre las mejores prácticas para una gestión de la OPSECU, como parte del plan de mejoramiento o direccionamiento estratégico que se puede plantear para la mejora continua y el logro de resultados hacia una gestión de calidad. 


\section{Cuadro 1. \\ Adaptación del Modelo de la EFQM para la Gestión de calidad de la OPSECU}

\begin{tabular}{cc}
\hline MODELO EFQM & Adaptación al contexto de la OPSECU \\
$\begin{array}{c}\text { Los Principios de la calidad total. } \\
\text { Descripción. (Gómez, 2017) }\end{array}$ & de la (FACFYM)
\end{tabular}

Orientación hacia los resultados: El éxito continuado depende de la satisfacción de las necesidades y expectativas de los distintos grupos de interés: clientes, proveedores, empleados, accionistas, sociedad.
Se obtiene conociendo las necesidades y expectativas de los grupos de interés (estudiantes, docentes, empleados, sociedad, entorno en general). Accionando (previo planeamiento estratégico), hacia el logro de los objetivos a corto, mediano y largo plazo; la gestión debe basarse en acciones coor dinadas y cónsonas con los fines y objetivos institucionales. Las exigencias de los grupos de interés deben ser atendidas mediante planes, programas y proyectos en pro de los resultados esperados.

La universidad debe definir claramente quienes son los clientes, ya que no es una empresa productora de bienes; considerando el derecho que tenemos todos a acceder a ella; la universidad por medio de la OPSECU (FSCFyM), satisfará las expectativas por medio de la labor académico-investigativa de cultura y de extensión, en beneficio de las comunidades pero también de sus componentes internos. Atendiendo los cambios provenientes de la innovación y la globalización. Estableciendo vínculos con su entorno mediante sus es tudiantes, docentes y empleados, a través de las actividades que realiza y al mismo tiempo promoviendo la ejecución de planes y proyectos orientados a la satisfacción de necesidades. La excelencia dependerá del equilibrio entre la eficiencia, la mejora continua y la satisfacción de necesidades (internas y externas) clientes; siendo estos los que se benefician de mación obtenida antes: expectativas y al fina: satisfacción
Gestión por procesos y hechos: la gestión por departamentos se debe complementar con la gestión por proceso, para adaptarse mejor a las necesidades

del cliente.

La gestión de la OPSECU (FACFyM), se debería complementar con las funciones de las demás oficinas de proyección social, y extensión cultural y universitaria de las demás escuelas (Estadística, Computación e Informática e Ingeniería Electrónica). Debido a que todas cumplen las mismas funciones "transmitir a la comunidad los conocimientos científicos, tecnológicos, culturales y humanistas, a través del trabajo de las cinco escuelas profesionales". (Paredes, et al., 2019) \}- Por lo tanto, la gestión por procesos, permitiría adaptarse a las necesidades que demanda el entorno (sociedad en general) y que dependen de la interrelación pero más aún del intercambio de información relacionado con el funcionamiento de estas oficinas; uno: para mejorar los procesos y hacer frente a las amenazas y aprovechar las oportunidades de un modo eficiente y dos: para la atención de las necesidades del entorno, as como de las exigencias de su estructura interna, con miras al fortalecimiento de sus capacidades.

Liderazgo y perseverancia en los objetivos:

- La dirección en el proceso hacia la Calidad Total, debe lograr que esta estrategia de gestión se despliegue a toda la organización, asumiendo el liderazgo del pro yecto para conseguir que se integre en la cultura de la organización.

- La dirección muestra en cada una de sus actuaciones su compromiso con la Calidad Total.

- Los directivos y demás clientes líderes deben facilitar todos los medios necesarios al resto de la organización
La tarea de los líderes es clave, con una importancia trascendental; en ese sentido en la OPSECU (FACFyM), los objetivos planteados se cumplirán en la medida que las personas que lideran desempeñen el rol de integradores y estén comprometidos con el desarrollo de la institución; además de constituirse en un ejemplo para el resto, ser capaces de establecer una dirección estratégica definida, que permita alinear a todas las personas involucradas hacia el logro de la excelencia en los propósitos, con ética y valores personales y organizacionales; todo en pro de reorientar las acciones hacia resultados de calidad. 


\section{Cont... Cuadro 1.}

Desarrollo e implicación de las personas: en este principio se establece el desarrollo del potencial de las personas, su implicación y participación en los procesos.
Las personas son la base para el funcionamiento óptimo de cualquier organización; en el caso de la OPSECU este es un aspecto de vital importancia, siendo que la función básica es la vinculación de la universidad con el entorno, esto es imposible realizarlo sin personas.

Para lograr la proyección social y extensión cultural y universitaria, deben existir tanto cantidad como calidad de personal, por sus roles y tareas a desempeñar. Por lo tanto se deben enfocar en la capacitación de cada uno, priorizando en el desarrollo personal y profesional que les brinde la oportunidad de crecer y especializarse, a modos de promover la implicación y participación eficiente y efectiva en los procesos; en correspondencia con la misión y visión de la institución, aunado a la naturaleza y función de la OPSECU. Para este fin es también de importancia delegar responsabilidades, promoviendo el compromiso y el trabajo en equipo.

La calidad total se consigue desafiando el "statu quo" y haciendo realidad el cambio, aprovechando el aprendizaje para crear innovación y oportunidad de mejora. Las decisiones a tomar y los proyectos a desarrollar deben derivarse de las necesidades de la organización. En el caso de la OPSECU, estos aspectos son los principales motores hacia el logro de la excelencia, es un proceso sistémico y cíclico, por lo tanto cada actividad que se lleve a cabo depende de la anterior para proseguir. En la OPSECU es importante resaltar la importancia de este ciclo puesto que para el logro de resultados óptimos en la gestión, deben participar todos los involucrados de forma interconectada.

Son importantes las relaciones con los proveedores directos, con el entorno, con las instituciones que comparten mismos fines, misión, visión y ubicación geográfica. La aplicación de este proceso permitirá a la OPSECU potenciar la conexión de la universidad con el entorno y sociedad en general; al mismo tiempo que se fortalecerá la institución por las experiencias, aportes enriquecedores, añadiendo valor tanto a las personas como a los procesos. La creación de vínculos con colaboradores potenciará a la institución con conocimientos, estrategias, servicios, lo cual será beneficioso y además recíproco; en la medida de la eficacia en las relaciones. El desarrollo de alianzas estratégicas, contribuye a crear valor agregado, confianza por parte del entorno, crecimiento, derrumbe de barreras, impacto social.

En este sentido la institución (Universidad Nacional Pedro Ruíz Gallo, Facultad de Ciencias Físicas y Matemáticas, Oficina de Proyección Social y Extensión Cultural y Universitaria; debe operar atendiendo a requisitos y normativa legal mínima, sobre RS-Responsabilidad Social; en relación con sus procesos de gestión. Esforzándose por dar respuesta a las expectativas de los grupos de interés en cuanto a RS. Este aspecto se desarrolla por el respeto e implementación de normativa y marcos legales, que van en beneficio de la sociedad en general, pero de la estructura interna también y por ende del personal que allí labora. Es entendida como la asunción de las obligaciones sociales, en un compromiso consecuente en su actuación, así como mostrar sentido del deber, la obligación y el cumplimiento de actividades individuales y sociales. (Medina, Franco, Torres, Velázquez, Valencia y Valencia, 2017; citando a: Villavicencio, 2013).)
Responsabilidad Social: La organización zación puede participar en iniciativas sociales. en ella. (Haug, 2017; Vilalta, 2011; citados por: Medina et al., 2017). Por lo antes dicho, los responsables de los servicios educativos, deben comprender y considerar el impacto que tienen los conocimientos sobre la sociedad; las escuelas y universidades deben manejarse de forma muy transparente, ya que su accionar repercute de 
manera muy significativa en la realidad circundante (sociedad, comunidades, regiones).

Al respecto, los hechos y producciones científicas (eventos, seminarios, congresos) que se llevan a cabo en los claustros universitarios son una clara demostración que la importancia de la universidad no radica solamente en las sesiones de clases, sino en todas estas actividades extracurriculares que se convierten en experiencias académica y socialmente enriquecedoras para los estudiantes y para el entorno que los rodea geográficamente. En este sentido Medina et al., (2017), opina que las instituciones universitarias tienen la oportunidad de potenciar el carácter bidireccional y dialógico del vínculo universidad-territorio, transformando a este en fuente y destino sistemáticos del conocimiento, gracias a la integración de los actores locales.

De igual forma, la extensión cultural y universitaria es una de las funciones definitorias de las universidades públicas latinoamericanas, herederas del movimiento de reforma universitaria ocurrido en el continente durante la primera mitad del siglo XX. No existe una definición única, ni el alcance de lo que significa específicamente "extensión universitaria"; por el contrario existen diferentes modos de entender lo que es, asociándose comúnmente con actividades de difusión cultural o artísticas. (Cano y Castro, 2016).

En ocasiones se la concibe a la extensión universitaria, como la acción de comunicar resultados de investigaciones, o también se le circunscribe al desarrollo de actividades de asistencia, asesoramiento técnico o transferencia tecnológica, en diferentes áreas del conocimiento. No muy pocas veces se le define como: "toda aquella actividad, que sin ser investigación o académica, la realiza la universidad en vinculación con organismos estatales, empresas, organizaciones sociales, comunidades, entre otras, todos estos, interlocutores no universitarios." (Cano et al., 2016).

En este punto, resulta oportuno resaltar el vínculo que si o si, debe existir entre la universidad y la sociedad, pues de esta manera se evidencia que la "Universidad Nacional Pedro Ruiz Gallo", debe cumplir una función social que influya y transforme su entorno, es decir, debe ser un auténtico factor de transformación social, y por lo tanto, según sea el fin que se proponga, aporte a la construcción de una sociedad de vanguardia. Dicho esto, para tal fin en cada facultad funciona una oficina de Proyección Social y Extensión Cultural y Universitaria (OPSECU), que se supone se encarga de prestar servicios y realizar actividades conducentes a la solución de problemáticas existentes en la comunidad; además de transmitir y trasferir los conocimientos científicos, tecnológicos, culturales y humanistas a través de la participación de las cinco escuelas profesionales. (Paredes et al., 2019).

En el caso de la Facultad de Ciencias Físicas y Matemáticas, la proyección social y extensión, al ser aspectos no considerados en el plan curricular de las escuelas profesionales, se produce una visión fragmentada de la realidad, se han aislado de su contexto social, a consecuencia de una enseñanza predominantemente teórica.

De acuerdo con Paredes, et al., (2019), la oficina brinda servicios de forma muy restringida, debido a la falta de presupuesto, lo que imposibilita la eficiencia de la gestión; 
convirtiendo a la FACFyM en un ente de carácter netamente formativo, pero sin el aspecto de interdisciplinariedad para los estudiantes, y a la OPSECU, como un departamento que no define situaciones vinculantes de la universidad con el entorno, debido a las fallas de su infraestructura.

\section{Percepciones sobre la Gestión de la Oficina de Proyección Social y Extensión Cultural y Universitaria, OPSECU. Direccionamiento estratégico para la mejora de la gestión.}

Con base en los resultados obtenidos, luego de la aplicación del cuestionario a la muestra seleccionada de docentes, estudiantes y egresados, con una escala tipo Lickert con 5 opciones de contestación que van como sigue: nunca, casi nunca, a veces, casi siempre y siempre. Se elabora la siguiente tabla (Tabla $\mathrm{N}^{\circ} 1$ ), que recoge en porcentajes por indicadores manejados, la información necesaria para caracterizar el tipo de gestión que se lleva a cabo en la institución objeto de estudio); la valoración asignada y seleccionada a cada uno de los indicadores, fueron los valores relativos más resaltantes o significativos; lo que permite inferir sobre la situación real de la gestión desarrollada en la Oficina de Proyección Social y Extensión Cultural y Universitaria (OPSECU) de la Facultad de Ciencias Físicas y Matemáticas de la Universidad Nacional "Pedro Ruíz Gallo".

\section{Tabla 1.}

\section{Percepciones sobre la gestión de la OPSECU}

\begin{tabular}{|c|c|c|c|c|c|c|}
\hline \multirow{3}{*}{$\begin{array}{c}\text { Indicadores que carac- } \\
\text { terizan la gestión de la } \\
\text { OPSECU - } \\
\text { FACFyM - UNPRG }\end{array}$} & \multicolumn{4}{|c|}{$\begin{array}{l}\text { Percepción de los participantes del cuestionario } \\
\text { (muestra) } \\
\text { Opción seleccionada con mayor \% }\end{array}$} & \multicolumn{2}{|c|}{$\begin{array}{l}\text { Resultados signi- } \\
\text { ficativos }\end{array}$} \\
\hline & \multirow{2}{*}{$\begin{array}{c}\text { Opciones } \\
\text { Escala Lickert }\end{array}$} & \multicolumn{3}{|c|}{ Muestra } & \multirow[b]{2}{*}{$\Sigma \%$} & \multirow[b]{2}{*}{$(\mathrm{pm}) \dot{\mathrm{X}}$} \\
\hline & & Docentes & Estudiantes & Egresados & & \\
\hline \multirow{5}{*}{$\begin{array}{l}\text { 1. Se promueve la integración } \\
\text { de diversas disciplinas y de } \\
\text { actores sociales externos en } \\
\text { los proyectos de investigación }\end{array}$} & Siempre & & & & - & - \\
\hline & Casi siempre & & & & - & - \\
\hline & A veces & & & & $\overline{-}$ & $\overline{-}$ \\
\hline & Casi Nunca & $33,3 \%$ & $48,2 \%$ & $48 \%$ & $129 \%$ & $43,1 \%$ \\
\hline & Nunca & & & & - & - \\
\hline \multirow{5}{*}{$\begin{array}{l}\text { 2. Los avances y resultados } \\
\text { de los proyectos de inves- } \\
\text { tigación que desarrollan en } \\
\text { la escuela profesional son } \\
\text { divulgados para la comunidad } \\
\text { universitaria }\end{array}$} & Siempre & & & & - & - \\
\hline & Casi Siempre & & & & - & - \\
\hline & A veces & $50 \%$ & & & $50 \%$ & $50 \%$ \\
\hline & Casi Nunca & & $28,9 \%$ & $40 \%$ & $68,9 \%$ & $34,45 \%$ \\
\hline & Nunca & & $17,4 \%$ & $26 \%$ & $43,4 \%$ & $21,7 \%$ \\
\hline
\end{tabular}




\section{Cont... Tabla 1.}

\begin{tabular}{|c|c|c|c|c|c|c|}
\hline \multirow{5}{*}{$\begin{array}{l}\text { 3. Se promueve la incorpo- } \\
\text { ración de descubrimientos } \\
\text { científicos y resultados de } \\
\text { investigaciones a fin de lograr } \\
\text { un impacto directo de lo inves- } \\
\text { tigado, en el desarrollo de la } \\
\text { sociedad }\end{array}$} & \multicolumn{4}{|l|}{ Siempre } & \multirow{3}{*}{$\begin{array}{l}- \\
- \\
118,7 \%\end{array}$} & \multirow{3}{*}{$\begin{array}{l}- \\
39.5 \%\end{array}$} \\
\hline & \multicolumn{4}{|l|}{ Casi siempre } & & \\
\hline & A veces & $46,7 \%$ & $44 \%$ & $28 \%$ & & \\
\hline & Casi Nunca & $46,7 \%$ & $41,3 \%$ & $48 \%$ & $136 \%$ & $45,3 \%$ \\
\hline & Nunca & & & & - & - \\
\hline \multirow{5}{*}{$\begin{array}{l}\text { 4. La ejecución de programas } \\
\text { y/o proyectos de proyección } \\
\text { social y extensión cultural y } \\
\text { universitaria responde a una } \\
\text { planificación previamente } \\
\text { definida }\end{array}$} & Siempre & $20 \%$ & $2,3 \%$ & $2 \%$ & - & - \\
\hline & \multicolumn{4}{|l|}{ Casi Siempre } & - & - \\
\hline & A veces & $43 \%$ & $40,8 \%$ & $28 \%$ & $111,8 \%$ & $37,2 \%$ \\
\hline & Casi Nunca & $20 \%$ & $27,1 \%$ & $40 \%$ & $87,1 \%$ & $29 \%$ \\
\hline & \multicolumn{4}{|l|}{ Nunca } & - & - \\
\hline \multirow{5}{*}{$\begin{array}{l}\text { 5. Se debe contar con } \\
\text { presupuesto para la imple- } \\
\text { mentación de programas y/o } \\
\text { proyectos en la OPSECU }\end{array}$} & Siempre & $36.7 \%$ & & & $36,7 \%$ & $36,7 \%$ \\
\hline & Casi Siempre & $30 \%$ & $22 \%$ & $4 \%$ & $56 \%$ & $18,6 \%$ \\
\hline & A veces & $33,3 \%$ & $44,5 \%$ & $26 \%$ & $103.8 \%$ & $34,6 \%$ \\
\hline & \multicolumn{4}{|l|}{ Casi Nunca } & - & - \\
\hline & Nunca & & & & - & - \\
\hline \multirow{5}{*}{$\begin{array}{l}\text { 6. La política de comunicación } \\
\text { (interna y externa) de la OP- } \\
\text { SECU, posibilita el fomento de } \\
\text { valores positivos y el posicio- } \\
\text { namiento institucional }\end{array}$} & \multicolumn{4}{|l|}{ Siempre } & - & - \\
\hline & Casi Siempre & $33,3 \%$ & $4,6 \%$ & & $37,9 \%$ & $18,95 \%$ \\
\hline & A veces & $30 \%$ & $47,7 \%$ & & $77.7 \%$ & $38,85 \%$ \\
\hline & Casi Nunca & $36,7 \%$ & $43,6 \%$ & & $80,3 \%$ & $40,15 \%$ \\
\hline & \multicolumn{4}{|l|}{ Nunca } & - & - \\
\hline \multirow{5}{*}{$\begin{array}{l}\text { 7. Se prioriza el diálogo para } \\
\text { mejorar los procesos internos } \\
\text { promoviendo la participación } \\
\text { de la comunidad universi- } \\
\text { taria , para la formulación } \\
\text { de estrategias y decisiones } \\
\text { institucionales. }\end{array}$} & \multicolumn{4}{|l|}{ Siempre } & - & - \\
\hline & Casi Siempre & $46,7 \%$ & $11,5 \%$ & & $58,2 \%$ & $29,1 \%$ \\
\hline & \multicolumn{4}{|l|}{ A veces } & - & - \\
\hline & Casi Nunca & $20 \%$ & $25,2 \%$ & $62 \%$ & $107,2 \%$ & $35.7 \%$ \\
\hline & Nunca & $10 \%$ & $11,5 \%$ & & $21.5 \%$ & $17.8 \%$ \\
\hline \multirow{5}{*}{$\begin{array}{l}\text { 8. Se favorece la transparen- } \\
\text { cia en la gestión, a través de } \\
\text { la disponibilidad de datos, } \\
\text { informes, reportes periódicos } \\
\text { de la gestión universitaria. }\end{array}$} & \multicolumn{4}{|l|}{ Siempre } & - & - \\
\hline & \multicolumn{4}{|l|}{ Casi Siempre } & - & - \\
\hline & \multicolumn{4}{|l|}{ A veces } & - & - \\
\hline & Casi Nunca & $20 \%$ & $27,1 \%$ & $58 \%$ & $105,1 \%$ & $35 \%$ \\
\hline & Nunca & $10 \%$ & $16,1 \%$ & $1,6 \%$ & $27.7 \%$ & $9.2 \%$ \\
\hline \multirow{5}{*}{$\begin{array}{c}\text { 9. El mejoramiento continuo } \\
\text { es una práctica característica } \\
\text { de la OPCYECU y de la } \\
\text { FACFyM }\end{array}$} & \multicolumn{4}{|l|}{ Siempre } & - & - \\
\hline & \multicolumn{4}{|l|}{ Casi Siempre } & - & - \\
\hline & \multicolumn{4}{|l|}{ A veces } & - & - \\
\hline & Casi Nunca & $20 \%$ & $14,7 \%$ & $46 \%$ & $80,7 \%$ & $26.9 \%$ \\
\hline & \multicolumn{4}{|l|}{ Nunca } & - & - \\
\hline
\end{tabular}

Fuente: Elaboracion propia (2020), a partir de Paredes (2019). 
El cuadro (Cuadro 2), muestra un resumen de las apreciaciones de los participantes (muestra seleccionada), sobre las preguntas 0 indicadores relacionados con la gestión que se lleva a cabo en la OPSECU, con un análisis, interpretación e indicación de los criterios a aplicar para el direccionamiento estratégico de la gestión.

\section{Cuadro 2}

\section{Indicadores de la gestión de la OPSECU. Análisis e interpretación}

\section{Indicadores que caracterizan la gestión de la OPSECU - FACFyM - UNPRG}

Percepción de los participantes Resultados más significativos $\mathrm{Pm}(\mathrm{X})$
Aplicación de los Criterios del Modelo EFQM (Ver figura:1)
1. Se promueve la integración de diversas disciplinas y de actores sociales externos en los proyectos de investigación
Casi nunca

$43,1 \%$

- Casi nunca se promueve la integración de diversas disciplinas y de actores sociales externos en los proyectos de investigación. La OPSECU debe promover la integración de la universidad y el entorno, para el desarrollo del conocimiento en pro del incremento de la calidad en la gestión y sus resultados. (Paredes, et al., 2019, citando a Ayala, 2012). (Aplicar Criterio 4. Alianzas y Recursos)

Los avances y resultados de los proyectos de investigación que desarrollan en la escuela profesional a veces, son divulgados para la comunidad universitaria. La OP-

2. Los avances y resultados de los proyectos de investigación que desarrollan en la escuela profesional son divulgados para la comunidad universitaria
A veces

$50 \%$ SECU debe manejar criterios de comunicación interna y externa que incluya la divulgación de los resultados de la actividad científica hacia los grupos de interés que hacen vida en la universidad, a modos de lograr el enriquecimiento con los nuevos aportes. (Paredes, et al., 2019, citando a González, 1996). (Aplicar Criterio 7: Resultados en personas)

Casi nunca se promueve la incorporación de descubrimientos científicos y resultados de investigaciones a fin de lograr un impacto directo en el desarrollo de la

3. Se promueve la incorporación de descubrimientos científicos y resultados de investigaciones a fin de lograr un impacto directo de lo investigado, en el desarrollo de la sociedad
Casi nunca $45,3 \%$ sociedad. Es necesario que por medio de la gestión de la OPSECU, se fortalezca la relación universidad-empresa y sociedad; para el logro de objetivos comunes, solución de problemáticas existentes y satisfacción de expectativas, (Paredes, et al., 2019). (Aplicar criterio:8 Resultados en la sociedad)

La ejecución de programas y/o proyectos de proyección social y extensión cultural y universitaria, a veces responde a una planificación previamente definida. Es

4. La ejecución de programas y/o proyectos de proyección social y extensión cultural y universitaria responde a una planificación previamente definida
A veces $37,2 \%$ imperativo que se implemente un plan operativo o estratégico para el funcionamiento coordinado y coherente de la OPSECU. La planificación es una herramienta que permite ejecutar acciones para lograr los cambios que se requieren, e impulsar las reformas estructurales necesarias. (Paredes, et., 2019; citando a Aguerrondo, 2007 y Duker, 1999). (Aplicar Criterio: 9. Resultados clave).

Siempre se debe contar con presupuesto para la implementación de programas y/o proyectos en la OPSECU. El desarrollo y ejecución de proyectos involucra

5. Se debe contar con presupuesto para la implementación de programas y/o proyectos en la OPSECU la utilización de recursos financieros, un adecuado financiamiento permite llevar a cabo estas actividades, tal como lo establece el artículo $63^{\circ}$, donde se afirma que:se asigna con carácter de intangible, un fondo especial para la proyección social y extensión cultural y universitaria. (Paredes, et al., 2019). (Aplicar criterio: 4 Alianzas y recursos). 


\section{Cuadro 2}

6. La política de comunicación (interna y externa) de la OPSECU, posibilita el fomento de valores positivos y el posicionamiento institucional

La política de comunicación (interna y externa) de la OPSECU, casi nunca posibilita el fomento de valores positivos y el posicionamiento institucional. El estatuto en su artículo $62^{\circ}$, establece: "Los programas de proyección social y extensión cultural y universitaria se difunden utilizando los medios de comunicación social de la universidad, del Estado y otros de los que pueda servirse". La OPSECU es la encargada de realizar las acciones destinadas a la proyección social y extensión cultural y universitaria, dirigida hacia la comunidad. Siendo evidente que la débil comunicación existente sea superada con esfuerzo de voluntades y liderazgo. (Paredes, et al., 2019). (Aplicar Criterio 2: Política y estrategia)

Casi nunca se da prioridad al diálogo para mejorar los procesos internos, promoviendo la participación de la comunidad universitaria, para la formulación de estrategias y decisiones institucionales. Es vital que la universidad desarrolle su potencial de comunicación,

7. Se prioriza el diálogo para mejorar los procesos internos, promoviendo la participación de la comunidad universitaria, para la formulación de estrategias y decisiones institucionales
Casi nunca $35,7 \%$
8. Se favorece la transparencia en la gestión, a través de la disponibilidad de datos, informes, reportes periódicos de la gestión universitaria estableciendo el diálogo permanente y valorando los aportes constructivos con visión innovadora. (Paredes, et al., 2019). Es una premisa que debe reinar en todos los procesos y actividades que se lleven a cabo, siendo la OPSECU canal para este fin, por ser intermediario entre los miembros de la comunidad universitaria y la universidad (Directivos). (Aplicar criterios, 1: Liderazgo; 2: Política y estrategias y 3 : Personas)

Casi nunca se favorece la transparencia en la gestión, a través de la disponibilidad de datos, informes, reportes periódicos de la gestión universitaria. Contar con información oportuna, veraz y constante es decisivo para el proceso de toma de decisiones...este proceso debe darse con total transparencia para asegurar una gestión de calidad. (Paredes, et al., 2019) (Aplicar criterio 1: Liderazgo)

El mejoramiento continuo casi nunca es una práctica característica de la OPSECU y de la FACFyM. Todos los actores involucrados deben esforzarse para lograr estándares adecuados de calidad; es necesario innovar y promover el compromiso institucional. "Las universi-

9. El mejoramiento continuo es una práctica característica de la OPSECU y de la FACFyM
Casi nunca $26,9 \%$ dades están llamadas a reorientar los mecanismos de mejora al interior de sus estructuras, ya que los esquemas convencionales distan hoy día, de las exigencias del mercado globalizado" (Cookson, 2012; citado por Hernández, Martínez y Rodríguez, 2017). (Aplicar el criterio 3: Personas y Criterio 5: Procesos).

\section{Conclusiones}

Uno de los rasgos característicos de las universidades públicas es el relacionado al financiamiento, el cual dependedeungobierno; principalmenteel nacional. Este aspecto influye de manera determinante en el funcionamiento de las mismas; impactando directamente en los resultados de las actividades que se desarrollan. Con este estudio se evidencia que en la OPSECU (Oficina de Proyección Social y extensión Cultural y Universitaria la deficiencia presupuestal incide en la baja calidad de la gestión que se lleva a cabo en esta oficina. Como consecuencia de lo anterior 
existe desatención de las demandas y exigencias que se plantean desde la sociedad; acarreando un impacto negativo y la desvinculación entre la universidad y el entorno; a su vez las comunidades no demuestran sentido de pertenencia hacia la institución.

Pese a que la Facultad de Ciencias Físicas y Matemáticas debe ser un ente promotor del desarrollo de la ciencia y la tecnología; de la cual deben surgir los servicios y conocimientos generados por los estudiantes de las cinco escuelas profesionales, esto no se ve reflejado en la comunidad local y regional. Esto se fundamenta en los resultados obtenidos, los que indican que la baja calidad de la gestión desarrollada en la OPSECU, se deriva de la falta de financiamiento, pero a su vez de la ausencia de un liderazgo y direccionamiento efectivo. El personal encargado de tomar decisiones y liderar todos los procesos, y de desarrollar la gestión en la OPSECU, requiere de modernizar e innovar las acciones en el aspecto de la proyección y la extensión universitaria de la UNPRG.

Al carecer de un sistema de gestión que permita dinamizar las actividades que se realizan, es prioritario la implantación de un mecanismo, tal como se sugiere por medio de la interpretación y orientaciones que se derivan del análisis del Modelo EFQM de excelencia, cuyos principios y criterios sirven como referencia de lo que se debe implementar en una primera etapa, para superar la difícil coyuntura en la que se encuentra. La misma se ve reflejada en la opinión negativa que se transmite desde el exterior de la institución, pero también desde los propios actores, que hacen vida en su estructura, (facultades, escuelas, otros departamentos) docentes, estudiantes, egresados y comunidad en general.
La OPSECU debe promover la integración de la universidad con el entorno, en pro del incremento en la reciprocidad de los resultados de sus acciones. Utilizar los medios disponibles para la divulgación de la información y de los resultados de las investigaciones que se realizan, tanto para la comunidad universitaria, como hacia el entorno y las comunidades locales, es un paso imprescindible para el restablecimiento de los vínculos y el fortalecimiento de la relación: universidad-empresasociedad; siendo ésta la forma idónea para gestionar eficientemente las soluciones a problemáticas existentes, y la satisfacción de exigencias.

Con un plan operativo de mejoramiento para la gestión de la proyección social y extensión cultural y universitaria, respaldado por los mecanismos del modelo EFQM, partiendo del compromiso de los líderes, directores, y de todos los involucrados, se podrán lograr los cambios e impulsar las reformas necesarias. La escasez de recursos y el bajo presupuesto, debe ser sustituida por una buena utilización de estos factores; un liderazgo asertivo debe asirse de una planificación, que tome en cuenta como principal obstáculo para una buena gestión, el presupuesto y el uso adecuado de los recursos disponibles.

Las estrategias comunicaciones, el diálogo bidireccional, la integración, la priorización de la participación de todos los grupos de interés, valorando los aportes constructivos, en la toma de decisiones institucionales, son la base para la reconstrucción de un liderazgo de vanguardia y una gestión de calidad que permita el posicionamiento de la OPSECU, la FACFyM de la UNPRG, en el entorno donde se desenvuelve como organización, o institución. Todo 
Gestión de calidad para la proyección socio-cultural y extensiónuniversitaria

Paredes López, Lilian Roxana; Curo Maquén, Luis Alberto; Carbajal Cornejo,

Katherine; Núñez Puse; Sonia Magali

lo anterior depende indefectiblemente en el esfuerzo y la voluntad de todos, no solamente de los responsables de la gestión. Imperante es innovar en las perspectivas para la mejora continua, siendo perseverantes en la búsqueda de la excelencia y mantenerla cuando se logren todos los objetivos planteados.

\section{Referencias Bibliográficas.}

Alvarado, L., Hernández, J; y Chumaceiro, A. (2010), Calidad de servicio en universidades públicas venezolanas. Revista Teorías, Enfoques y Aplicaciones en las Ciencias Sociales - TEACs, 2(4), 45-58. Universidad Centroccidental Lisandro Alvarado (UCLA).

Alvarado P, Lisandro J; Izaguirre D, Fridzia (2015), Calidad del servicio en universidades públicas mexicanas: perspectivas de análisis desde las dimensiones. En Revista Pensamiento y Gestión. Universidad del Norte, 95-118, 2015.

Cano M, Agustín y Castro V, Diego (2016), La extensión Universitaria en la Transformación de la Educación Superior. El caso de Uruguay. En Revista Andamios, Vol.13, $\mathrm{N}^{\circ} 31$, pp: 313-337.

Castillo P, Carlos; Mercado I, Carmelo; Prado R, Camilo y Soto G, Federico (2014), Normativas EN-9100 e ISO9000 en el sector aeroespacial español. En Revista Venezolana de Gerencia, año 19, N $^{\circ} 67$, Venezuela, Centro de Estudios de la Empresa, Universidad del Zulia. pp. 410-434.

Fontalvo Tomás J; y De La Hoz, Efraín J. (2018), Diseño e Implementación de un Sistema de Gestión de la Calidad ISO 9001:2015 en una Universidad Colombiana. En Revista Formación Universitaria. Vol. 11/ (1), 35-44 (2018). Cartagena, Colombia.
Gómez-Vargas, Juan Carlos (2017), El Modelo EFQM. Los principios de la Calidad Total. Los Criterios del modelo EFQM y La Evaluación". Disponible en: https://www.researchgate.net/ publication/324544263 El Modelo EFQM Los Principios de la Calidad Total los Criterios del Modelo EFQM y la Evaluación. Fecha de consulta: 01.03.2020.

Hernández, Hugo; Martínez, David; y Rodríguez, Jorge (2017), Gestión de la calidad aplicada en el mejoramiento del sector universitario. En Revista Espacios, Vol. 38, N²0. Pág. 29.

Martínez Clares, Pilar; Pérez Cusó, Francisco Javier; y Martínez Juárez, Miriam (2016), Aplicación de los modelos de gestión de calidad a la tutoría universitaria En Revista Complutense de Educación. 29 (3) 2018, pp: 633-649.

Medina Peña, Rolando; Franco Gómez, María del C; Torres Barreiro, Leonadis; Velázquez Rodríguez, Kerslin; Valencia Vera, María Alexandra; Valencia Vera, Ana Leonor, (2017), La responsabilidad social universitaria en la actual sociedad del conocimiento. Un acercamiento necesario. En Revista Medisur, Vol. 15, Nㅜ 6, pp: 786-791.

Paredes López, Lilian Roxana; y Curo Maquén, Luís Alberto, (2019), Propuesta de gestión de la proyección social y extensión cultural y universitaria en la formación integral e interdisciplinaria del estudiante de la Facultad de Ciencias Físicas y Matemáticas-UNPRG. Tesis presentada para obtener el grado académico de Doctor en Ciencias de la Educación. Universidad Nacional Pedro Ruíz Gallo. Escuela de Posgrado. Lambayeque - Perú.

Pulido Rocatagliata, Sergio y Espinoza Díaz, Oscar (2018), Aseguramiento 
de la calidad en educación superior de Chile: Alcance., implicaciones y aspectos críticos. En Revista Venezolana de Gerencia, Año 23, Edición Especial $\mathrm{N}^{\circ}$ 1. 2018.
Venezuela, Centro de Estudios de la Empresa. Universidad del Zulia. pp: 238-255.

- Esta obra está bajo una licencia de Creative Commons Reconocimiento-NoComercialCompartirlgual 3.0 Unported. http://creativecommons.org/licenses/by-nc-sa/3.0/deed.es_ES 